\title{
La custodia compartida: ¿un paso más hacia la igualdad de género?
}

\section{Montse Solsona y Marc Ajenjo, Centre d’Estudis Demogràfics y Universitat Autònoma de Barcelona}

Los datos publicados por el Instituto Nacional de Estadística (INE) indican que desde 2011 ha habido una gran estabilidad en la cifra de divorcios: en torno a los 100 mil anuales, la mitad de los cuales con hijos/as menores de edad. Sin embargo, en el mismo período se ha producido un notable incremento de la custodia compartida en detrimento del paradigma anterior de custodia exclusiva de la madre con un régimen de visitas para el padre. Así, entre 2011 y 2016, la custodia compartida ha pasado del 12\% al 28\% de los divorcios con hijos/as menores. Este crecimiento, liderado por las comunidades autónomas que tienen una legislación favorable al respecto, refleja cambios sociales de mayor alcance relacionados con la igualdad de género dentro y fuera del hogar.

El análisis de las series del INE, sumado a un estudio inédito de todas las sentencias de divorcio y también de las rupturas de parejas de hecho no incluidas en las estadísticas del INE de los juzgados de familia de la ciudad de Barcelona del año 2014 -5.894 sentencias-, ilustran que la custodia compartida pactada significa, al menos sobre el papel, equidad entre el padre y la madre, tanto en el cuidado de los niños/as (residencia alternada) como en la participación en sus gastos.

A partir de estos resultados, cabe preguntarse si sería adecuado tras la separación de los progenitores otorgar por defecto la custodia compartida de los hijos/as. La respuesta no puede ser más clara: No. La custodia compartida debería ser la secuencia lógica de un pacto que reflejara una práctica anterior a la ruptura de coparentalidad en equidad, la cual a día de hoy todavía no es mayoritaria.

\section{LAS DOS ESPAÑAS}

El incremento de la custodia compartida entre 2011 y 2016 en España, se ha producido exclusivamente en detrimento de la custodia exclusiva de madre, dado que la exclusiva de padre se ha mantenido en torno al $5 \%$. Aunque la custodia compartida ha crecido en todas las comunidades autónomas, un ritmo muy desigual ha acentuado las diferencias iniciales (ver Figura 1). Las comunidades con porcentajes más elevados de custodia compartida y claramente por encima del promedio español son Islas Baleares, Cataluña, Valencia, Aragón, País Vasco, Navarra y la Rioja. En sólo cinco años -de 2011 a 2016-, todas ellas han añadido unos veinte puntos porcentuales a la prevalencia de la custodia compartida. Tal y como muestran los dos mapas de la Figura 2, en el año 2016 las comunidades con más custodia compartida, con la única excepción de Islas Baleares y la Rioja, coinciden con aquellas que tienen una legislación propia que promueve la coparentalidad después de un divorcio. En un estudio reciente hemos demostrado que, además de la importancia de la legislación, el segundo factor explicativo de la custodia compartida es la existencia de relaciones de género más igualitarias en el seno de la pareja y la sociedad en general, especialmente en la distribución del trabajo productivo y de cuidado (Solsona, Spijker y Ajenjo, 2017).

FIGURA 1. Evolución de la custodia compartida por comunidades autónomas. 2011-2016 (en \% sobre el total de custodias)

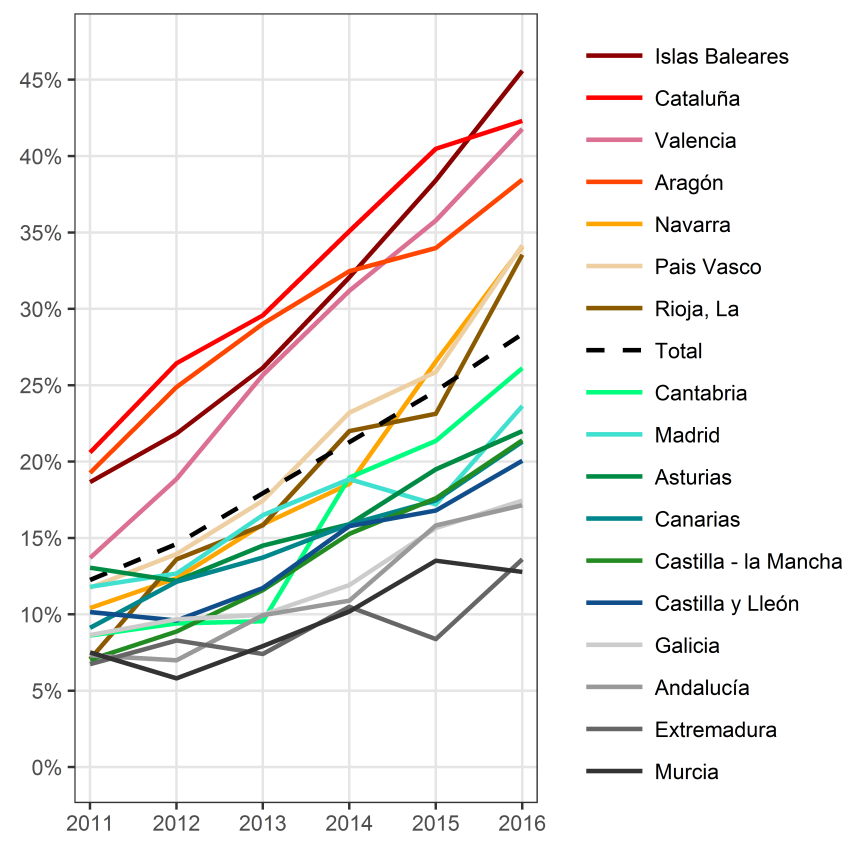

Fuente: Elaboración propia a partir de los microdatos de las Estadísticas de divorcios del INE. 


\section{IMPLICACIONES PARA LA DEMOGRAFÍA}

La custodia compartida tiene implicaciones directas en la demografía. Debemos tener en cuenta que un poco más de la mitad de las parejas divorciadas entre 2011 y 2016 tenían hijos/as, en conjunto unos 480 mil menores de 18 años, para los que se tenía que decidir sobre el tipo de custodia, siendo cerca de 100 mil a los que se otorgó la custodia compartida. Aunque estos datos no permiten precisar

FIGURA 2. Leyes autonómicas y custodia compartida por comunidades en 2016 (en \% sobre el total de custodias)
Leyes autonómicas

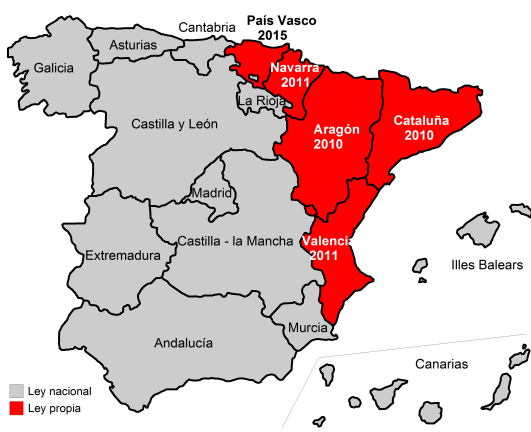

Custodia compartida, 2016

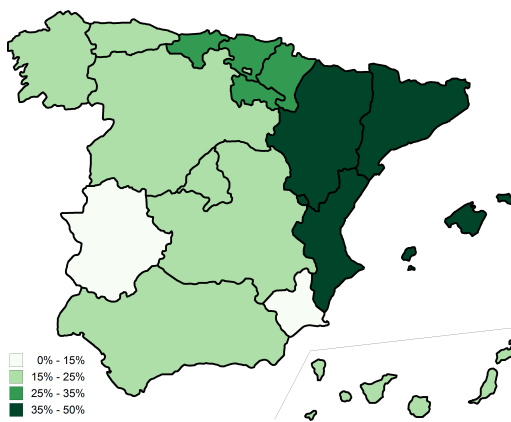

Fuente: Elaboración propia a partir de los microdatos de las Estadísticas de divorcios del INE.

cuántos menores de 18 años hay actualmente que vivan en residencia alternada, la encuesta Health Behaviour in School-aged Children (Moreno et al. 2016) muestra que, en 2014, alrededor de un 5\% de jóvenes comprendidos entre 11 y 16 años viven en régimen de custodia compartida. Con un sencillo cálculo podemos estimar que en 2014 había en España casi 150 mil adolescentes viviendo a partes iguales en dos hogares. Así, contrariamente a lo que nos informan los censos de población, que adjudican por imperativo legal a cada persona un solo domicilio, hay una parte nada despreciable de la población joven que tiene una doble residencia.

Asimismo, puesto que las estadísticas oficiales del INE sobre separaciones y divorcios no incluyen las rupturas de parejas de hecho, un colectivo que como veremos a continuación es cada vez más numeroso y además de características bastante diferenciadas, es muy conveniente que en breve se elabore una nueva estadística que las incluya.

\section{¿DE QUÉ HABLAMOS CUANDO HABLAMOS DE CUSTODIA COMPARTIDA?}

Veámoslo en un estudio de caso para la ciudad de Barcelona, donde analizamos la custodia compartida como hecho jurídico a partir de las 5.894 sentencias dictadas en los juzgados de familia en 2014. Al analizar directamente las sentencias, hemos podido incluir por primera vez, además de las rupturas matrimoniales, los procesos judiciales de parejas de hecho con hijos/as que se separan, ya que ambos siguen el mismo procedimiento legal. Así, hemos podido constatar que del total de pro- cesos judiciales en los que se dirime la custodia de menores, un $37 \%$ corresponden a parejas de hecho.

Los principales resultados de nuestra investigación permiten desmentir dos mitos. El primero hace referencia a la banalización de la custodia compartida, hipótesis planteada en varias ocasiones por profesionales jurídicos en 2014. Su impresión era que, en Barcelona, atendiendo a los criterios establecidos por la Ley Catalana de 2010 -experiencia de cuidado conjunta antes de la ruptura de la unión, capacidad de cada progenitor para hacerse cargo de las tareas parentales y actitud de ambos para cooperar en todo momento, entre otros-, se estaban otorgando más guardas compartidas de las que corresponderían. Los resultados, sin embargo, no apuntan en esta línea, en tanto la custodia compartida otorgada es menos frecuente que la corresponsabilidad parental en el cuidado de los hijos/as entre las parejas no divorciadas con hijos/as menores. En efecto, en 2014 en Barcelona el porcentaje de las parejas que una vez rota la unión lograban una custodia compartida -un 25,3\%- eran inferior al de las parejas con hijos/as menores de 18 años -30,8\%-que según la Encuesta de Usos del Tiempo 2009-2010 compartían las tareas domésticas y de cuidado en condiciones de igualdad. Aunque no podemos deducir un efecto de causa-consecuencia, los indicios apuntan a que en el caso de Barcelona y, por extensión de Cataluña, el criterio de coparentalidad antes del divorcio parece bastante razonable.

El segundo mito se refiere a la situación de las parejas de hecho. Todo parece indicar que las personas que forman una unión sin papeles, en principio más acostumbradas al pacto, deberían resolver su ruptura sin la intervención de un juez y mostrar un mayor número de custodias compartidas. Y, al menos para las parejas de hecho que pasan por los juzgados, esto no es así. Estas muestran un mayor grado de conflicto -en términos judiciales seguir un procedimiento contencioso en el que el juez tiene la última palabra- que las parejas casadas y, además, tal y como muestra la Figura 3, tienen menos custodias compartidas.

\section{EL PACTO ENTRE LOS CÓNYUGES ES CRU- CIAL}

Además del tipo de unión, la Figura 3 muestra otros factores que favorecen el paradigma emergente de la custodia compartida. Uno de los más relevantes es, sin duda, el pacto entre los progenitores -en términos judiciales seguir un procedimiento de mutuo acuerdo. Es tres veces más probable una custodia compartida cuando es la pareja quien pacta los términos de la separación, que cuando es el juez quien los determina.

El lugar de origen de la pareja también tiene un fuerte poder explicativo. 
Cuando ambos progenitores son españoles, la propensión a la custodia compartida es cuatro veces más elevada que cuando ambos son extranjeros. Ahora bien, debemos destacar también que es más importante el lugar de nacimiento de él que el de ella, en tanto que se otorgan (o se pactan) más custodias compartidas cuando el padre es español y la madre extranjera, que en la situación inversa.

Estos resultados para la ciudad de Barcelona coinciden con la investigación sobre los factores determinantes de la custodia compartida -a partir de las estadísticas del INE- para el conjunto de Cataluña y el resto de España (Solsona y Spijker, 2016). En el estudio de Barcelona hemos podido ir más lejos, analizando el efecto de otras variables (como el tipo de unión que ya hemos visto, la edad de los hijos/as y la clase social) no incluidas por el INE.

FIGURA 3. Custodia compartida según diferentes variables. Ciudad de Barcelona, 2014 (en \% sobre el total de custodias)

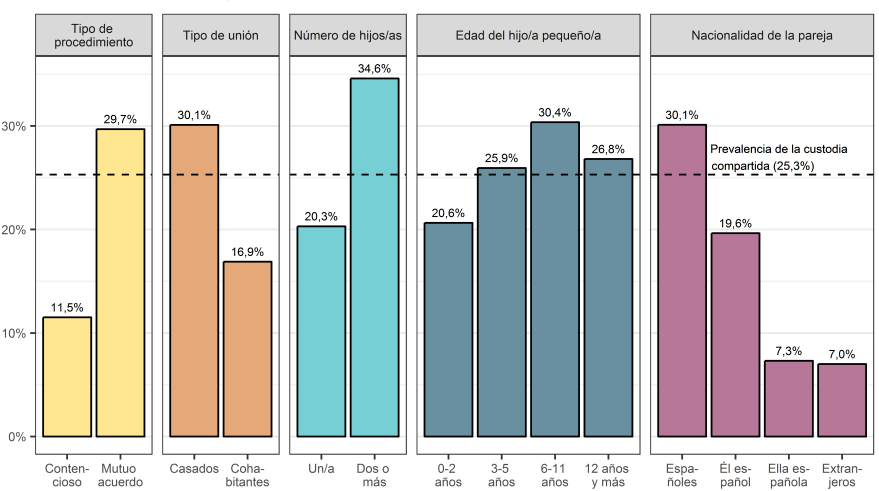

Fuente: Elaboración propia a partir de las sentencias de todos los juzgados de familia de Barcelona.

La custodia compartida es más frecuente cuando el hijo/a menor tiene entre 6 y 11 años, lo cual parece reflejar la opinión de diferentes operadores jurídicos vinculados a la infancia poco partidarios de la itinerancia de los menores de 6 años entre dos hogares. A partir de los 12 años la doble residencia decae.

Para Barcelona hemos podido analizar el efecto de la clase social únicamente para los procesos judiciales de mutuo acuerdo -motivo por el cual no se ha incluido en la Figura 3. Y hemos podido comprobar que existe una clara relación positiva entre renta familiar y custodia compartida. Aquellos que tienen rentas más altas acuerdan en un $42 \%$ de los casos la custodia compartida, mientras en las rentas más bajas la prevalencia es sólo del 24\%, y del 32\% entre las clases medias. Esta constatación nos permite afirmar que la falta de recursos tiene un efecto negativo, no sólo en la propensión a divorciarse, sino también en la posibilidad de mantener dos hogares en las mismas condiciones.

Entre todos los factores estudiados el pacto tiene una importancia capital porque forma parte de la gestión del propio proceso de ruptura, que per se ya supone un cierto conflicto. El pacto es un principio que recoge la ley catalana de 2010, al proporcionar un instrumento -el Plan de Parentalidad- diseñado para promover el acuerdo entre las partes (Solsona, Brullet y Spijker, 2014) y que, entre otras cosas, contempla el régimen de estancias de los hijos/as en casa de cada progenitor. Es sabido que el pacto es crucial no sólo para la coparentalidad, sino también para los resultados académicos y para el bienestar de los hijos/as. En un estudio reciente, Escarpa (2017) demuestra que los hijos/as de un divorcio sin conflicto obtienen mejores resultados en los estudios que los que viven en un hogar biparental. En este estudio de Barcelona hemos analizado cómo se resuelve la distribución del tiempo de cuidado y la financiación de los gastos de los niños/as entre la madre y el padre, dos elementos esenciales para garantizar su bienestar.

\section{TIEMPO Y DINERO EN EQUIDAD DE GÉNERO}

Al menos sobre el papel, la custodia compartida significa equidad entre el padre y la madre tanto en tiempo de cuidado como en los gastos de los niños/as.

La Figura 4 muestra el porcentaje de noches que, según las sentencias, los menores de 18 años duermen en casa de su padre. Si el acuerdo es consecuencia de un pacto -procedimiento de mutuo acuerdo- la igualdad en el tiempo de cuidado es absoluta: duermen la mitad de las noches en casa de cada uno de los progenitores. Aunque en menor medida, esta igualdad también la podemos ver en las custodias compartidas provenientes de un proceso contencioso. La mayor diferencia entre el mutuo acuerdo y el contencioso, que se acentúa aún más en las custodias exclusivas de madre, es el desequilibrio entre los días festivos -fines de semana y vacaciones escolares, en general-y las noches del período escolar. Una diferencia que tiene una importancia

FIGURA 4. Residencia alternada de hijos e hijas en días de escuela y festivos* según el tipo de custodia y de procedimiento (mutuo acuerdo o contencioso). Barcelona, 2014 (\% de pernoctas anuales con el padre)

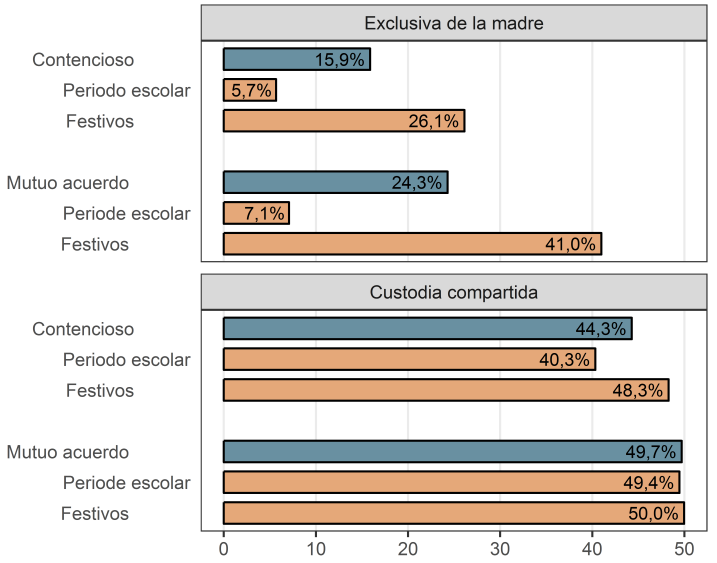

*Los días festivos incluyen fines de semana, vacaciones de Navidad, Semana Santa y verano.

Fuente: Elaboración propia a partir de las sentencias de todos los juzgados de familia de Barcelona. 
capital en tanto que los días de escuela son también días de actividad laboral, dificultando a la madre la conciliación de los ámbitos productivo y reproductivo.

La misma lógica se da en el pago de las pensiones (Figura 5). En el 88\% de las custodias compartidas que provienen de un mutuo acuerdo, o bien ninguno de los progenitores paga pensión alguna -lo que es equivalente a que cada uno pague los gastos de los hijos cuando convive con ellos- o bien los dos hacen una aportación a modo de pensión. En este último caso se trata de una cantidad ligeramente más elevada para los hombres, muy probablemente como consecuencia de un mayor poder adquisitivo. Cuando la custodia compartida proviene de un contencioso en el 50\% de los casos paga solamente el padre, lo cual la sitúa en un punto intermedio entre la custodia compartida pactada y la custodia exclusiva de la madre.

Por otra parte, tanto la Figura 4 como la Figura 5 muestran que la custodia exclusiva de la madre encarna el paradigma anterior, en el

FIGURA 5. Pensiones de alimentos de los hijos/as según el tipo de custodia y de procedimiento. Barcelona, 2014 (en \% de quién paga).

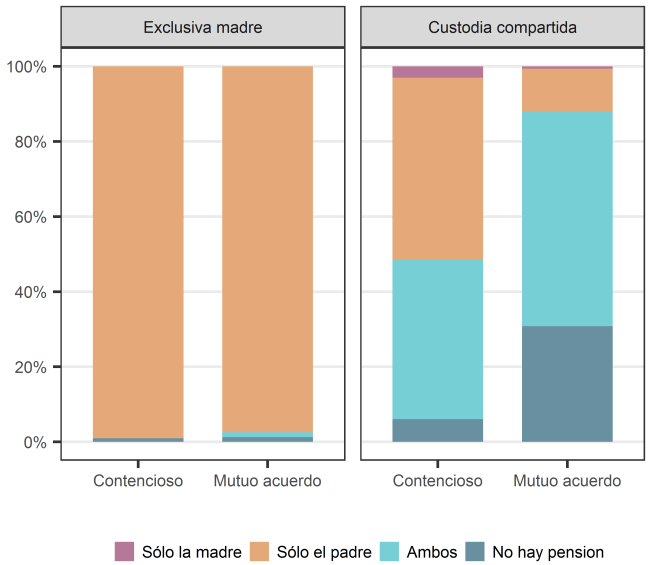

Fuente: Elaboración propia a partir de las sentencias de todos los juzgados de familia de Barcelona.

\section{Referencias bibliográficas}

Ajenjo, M y García-Román, J (2014) «Cambios en el uso del tiempo de las parejas ¿Estamos en el camino hacia una mayor igualdad?», Revista Internacional de Sociología, 72 (2): 453-476.

Escarpa, S (2017) «Effects of PostDivorce Parental Conflict on Children's educational achievement», REIS, 158 : 41-58.

Moreno, C; Ramos, P; Rivera, F et al (2016) Informe técnico de los resultados obtenidos por el Estudio "Health Behaviour in School-aged Children (HBSC) 2014" en España. Madrid: Ministerio de Sanidad, Servicios Sociales e Igualdad.

Solsona, M; Brullet, Cy Spijker, J (2014) «Coparentalitat i custòdia compartida a Catalunya", Documents d'Anàlisi Geogràfica, 60 (2): $387-415$
Solsona, My Spijker, J (2016) «Does the 2010 Catalan Civil Code affect trends in joint physical custody? A comparison between Catalonia and the rest of Spain», Population, 71 (2): 297-324.

Solsona, M; Spijker, Jy Ajenjo, M (2017) «Calidoscopio de la custodia compartida en España» en Becerril, D \& Venegas, M. (Coor.) La custodia compartida en España. Madrid: Dykinson, 45-72.

Cita

Solsona, Montse y Ajenjo, Marc (2017) "La custodia compartida: ¿un paso más hacia la igualdad de género?", Perspectives Demogràfiques, 8: 1-4.

Editores:

Andreu Domingo y Albert Esteve

que confluyen las desigualdades en el ejercicio de la maternidad y la paternidad en tres dimensiones: el tiempo de cuidado, la conciliación entre trabajo y familia y los gastos de los niños/as.

\section{LA CUSTODIA COMPARTIDA ES UN AVAN- CE HACIA LA EQUIDAD, PERO...}

Hasta aquí hemos estudiado los acuerdos legales. La correspondencia entre los acuerdos y las prácticas parentales dependerá, a nivel individual, de la práctica de la corresponsabilidad antes de la ruptura, y a nivel colectivo de la solidez del cambio social con respecto a las nuevas paternidades. A nuestro entender, la sostenibilidad en el tiempo de los acuerdos y de las prácticas de coparentalidad en equidad traerá consigo que las nuevas generaciones de niños y niñas estén habituadas al rol de cuidado intercambiable entre progenitores, lo cual redundará en la adquisición de valores de equidad a través de su propia experiencia de socialización.

La custodia compartida debe ser vista como un avance si se trata de un reflejo, tal como dice la ley catalana, de la corresponsabilidad previa a la disolución de la pareja. Por tanto, la respuesta legislativa más adecuada no debe consistir en imponer la custodia compartida, sino que debe tener en cuenta las condiciones de cuidado previas a la ruptura y dejar que el aumento progresivo de las parejas igualitarias que se está produciendo (Ajenjo y García- Román, 2014) conduzca, en caso de divorcio, a un aumento de las custodias compartidas.

Ahora bien, si la custodia compartida fuera otorgada de manera indiscriminada podría agudizarse el conflicto entre los progenitores, y tal vez las desigualdades entre ellos. Por lo tanto, la aplicación de una legislación que no tenga en cuenta la peculiaridad de cada caso, su historia, y que obvie que las medidas no pueden ser impuestas en un contexto donde las desigualdades entre hombres y mujeres son plenamente vigentes, puede ser claramente contraproducente.

\section{Correspondencia dirigida a:}

Montse Solsona

msolsona@ced.uab.es

\section{Créditos}

Diseño de la investigación: Montse Solsona, Cristina Brullet y Marc Ajenjo

Trabajo de campo y base de datos: Amalia Gómez Casillas

Gráficos: Anna Turu

Maquetación

Xavier Ruiz Vilchez

Agradecimientos

A Montserrat Tur, al personal de los Juzgados de Familia de la Ciudad de la Justicia de Barcelona, y a todo el equipo de trabajo de campo.

\section{Enlace url}

http://ced.uab.es/es/difusion/butlletiperspectives-demografiques

Contacto

Centre d'Estudis Demogràfics. Calle de Ca n'Altayó, Edificio E2 Universitat Autònoma de Barcelona o8193 Bellaterra / Barcelona España Teléfono: +34 935813060 E-mail: demog@ced.uab.es Web: http://ced.uab.es/es/

\section{UAB Universitat Autònoma de Barcelona}

A50 